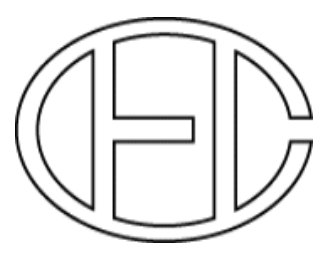

\title{
Best current practices - Recommendations on electronic information communication (2002)
}

\author{
Endorsed by the IMU Executive Committee on April 13, 2002 in its 69th session in Paris, France
}

Communication of mathematical research and scholarship is undergoing profound change as new technology creates new ways to disseminate and access the literature. More than technology is changing, however; the culture and practices of those who create, disseminate, and archive the mathematical literature are changing as well. For the sake of present and future mathematicians, we should shape those changes to make them suit the needs of the discipline.

For this reason, we have identified a number of "best practices" for those involved with the mathematical literature - mathematicians, librarians, and publishers. Many of these are practices that apply to other academic disciplines as well. Although we focus primarily on mathematics, we recognize that we can learn from each other as we move forward, and that no single discipline should act in isolation.

Our advice is meant to guide practice as it changes rather than to set forth a collection of firm rules and admonitions. The recommendations concern all forms of scholarly publishing and do not promote any particular form. Indeed, the authors of this document hold many differing views on the future of scholarly publishing. The common principle used to formulate our recommendations is that those who write, disseminate, and store mathematical literature should act in ways that serve the interests of mathematics, first and foremost.

This is advice that is meant to ease the transition in scholarly communication for present mathematicians. Most importantly, however, it is advice aimed at protecting mathematicians in the future.

\section{For mathematicians}

1. Structure And Format. Logically structured documents correctly reflect the content of a mathematician's work, setting forth results, arguments, and explanations to make them understandable to readers. But a logical structure also makes it possible to retrieve and eventually to update the document. Identifying the constituent parts of an electronic document is essential in order to move from one format to another without human intervention. Authoring documents should be more than setting down mathematical research in a pleasing format.
Authors are encouraged to provide the structure necessary to use their documents now and in the future. The aim is to create a master file from which the various other formats can be derived. [In mathematics, ${ }_{\mathrm{AT}} \mathrm{XX}$ is a congenial and accessible way to give documents some structure without adding unreasonable burdens on the author.]

2. Linking AND ENRICHMENT. An electronic publication can offer much more than a print publication. Electronic publication gives the user the ability to move effortlessly among the various parts of a paper or even from one paper to another. In order to make this possible, however, someone must add the necessary information to establish links in the electronic version.

Adding links is easier when authors provide the information necessary to establish them. [Correct crossreferencing and citation in $\mathrm{AT}_{\mathrm{EX}} \mathrm{X}$ transforms readily into hyperlinks, yielding enriched electronic versions of one's work. Hyperlinks may be used in PDF files as well.]

Moreover, electronic publication is not restricted by the constraints of the traditional print medium. This provides an opportunity to detail material that might otherwise be dismissed as "well known" and to add explanatory appendices. A little less easily, whenever appropriate, one may include graphic enhancements, animations, extensive data, tools to analyze that data, or even active examples that may be varied by the reader.

3. Versions. Online publication can lead to severe problems in citation, because the posted paper can be updated continuously until it bears little resemblance to the original, as an author corrects, adds, and deletes material without indicating that changes were made. As the mathematical literature grows, references to non-existent papers and results will eventually jeopardize its coherence. 


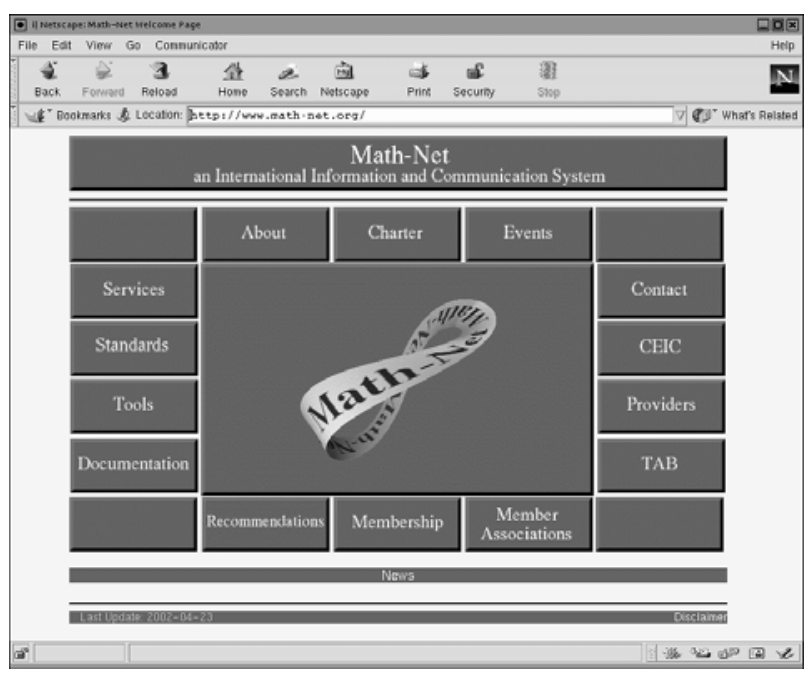

To avoid this problem, papers that have achieved a sufficiently final state should be stored in an immutable form. This includes any paper to which others may make reference, whether published in refereed journals or posted as a preprint. If revisions subsequently are necessary, each released version should be clearly labeled with its own version number and old versions should remain available.

4. Personal Homepages. Mathematical communication is more than merely posting or publishing papers. Information about the mathematical community and its activities is valuable to all mathematicians, and it is now easier than ever to circulate and to find such material.

Mathematicians are encouraged to have their own homepage. Ideally, basic data on such a page (or on a "secondary" homepage) should be presented in standard form to allow ready automatic compilation into databases.

[Material found at http://www.math-net.org/ Math-Net_Page_Help.html describes the Math-Net project, which provides standardized homepages for departments and institutes.]

5. Personal Collected Works. Mathematics ages slowly. Access to older literature is important for most mathematicians, and yet much of the older literature is likely to remain unavailable in electronic form in the immediate future. Mathematicians can change that by taking collective action.

Whenever legally and technically possible, mathematicians are encouraged to scan their old (pre- $\mathrm{T}_{\mathrm{E}} \mathrm{X}$ ) papers and post them on their homepages, making their "collected work" readily available to all. This relatively small effort on the part of every mathematician will provide enormous benefit to the entire community.

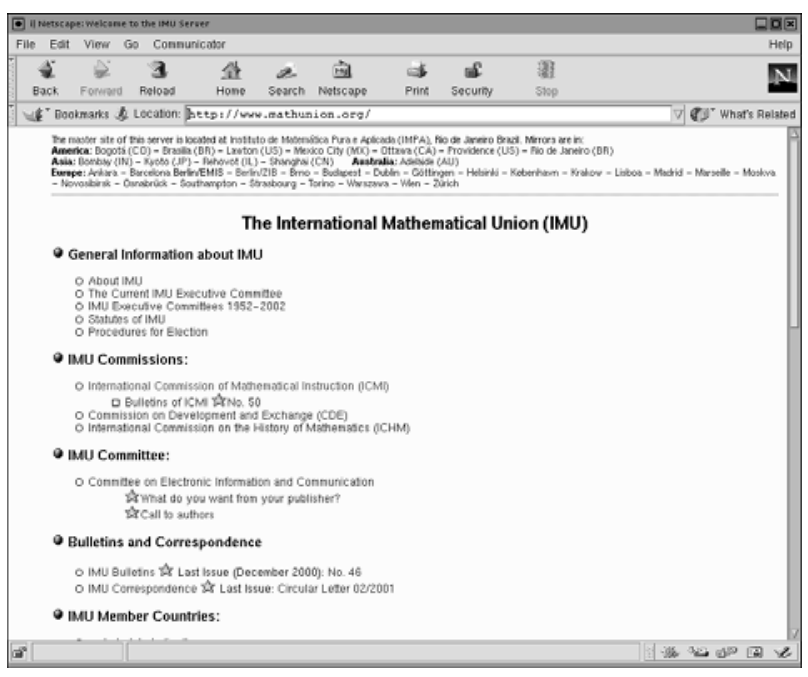

The Call to Mathematicians found at http://www. mathunion.org provides further information.

6. Preprints And Archives. Mathematical writing is ineffective if it is not communicated. A generation ago, the photocopier made it easy to send preprints to one's peers. Today, as a substitute, we have departmental servers, homepages, and public archives. [The arXiv (http://www.arxiv.org/) is one prominent example.]

It is a good practice to place one's preprints both on a homepage and in an appropriate archive. Either copy serves to communicate the mathematics to one's peers, but the public archive will make it more likely that others can reference your work in the future.

7. Copyright. While copyright is a complex subject that is far removed from mathematics, copyright law and policy can profoundly affect the ways in which mathematics is disseminated and used. Copyright is important for mathematicians.

Authors should be aware of the basic principles of copyright law and custom. Decisions about copyright for one's own work should be made thoughtfully.

The material found at http://www.ceic.math.ca/ serves as a good reference.

\section{For librarians and mathematicians}

8. Journal Prices And Policy. Libraries have limited budgets, which often grow more slowly than the prices of journals, forcing libraries to cancel subscriptions. The cumulative effect of cancellations goes beyond individual institutions because it shifts costs to an ever smaller number of subscribers, accelerating the process of price increase and cancellation. Journal prices matter to all mathematicians. 


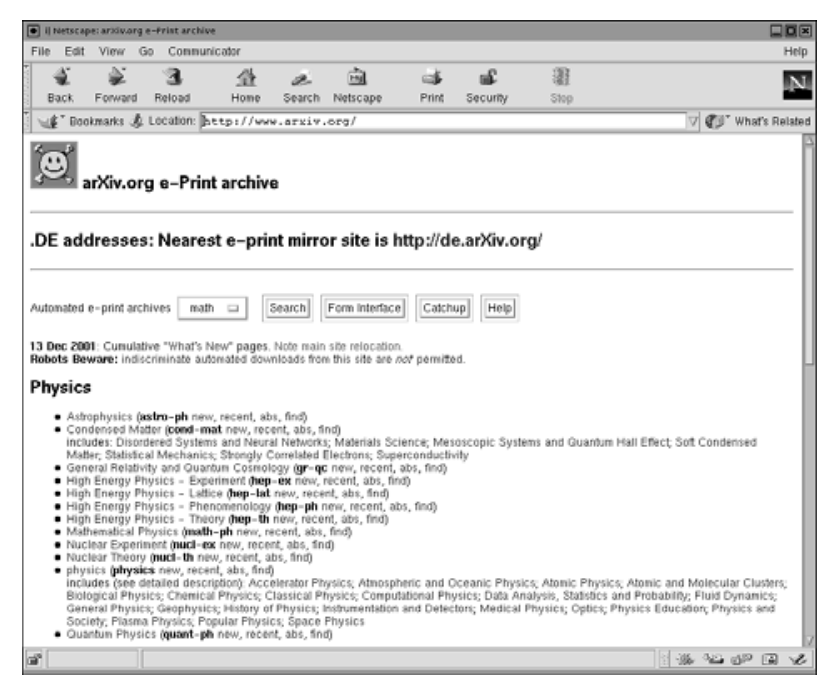

When deciding where to submit a paper an author may choose to be aware of a journal's standing and impact, but an author also should take account of a journal's price (as well as its general policies, including archiving). In addition, one might consider a journal's price and policies when considering whether to referee or serve on an editorial board.

9. Validation. Publication and peer review processes are increasingly detached. The emergence of overlay journals, archival preprint servers, and other new structures of publication raise new and pressing questions about the appropriate forms of validation. These are important issues for all scholarship, but even more important for mathematics since it is essential to know which parts of the mathematical literature are valid.

Both mathematicians and decision makers need to be alert to the distinction between posting and providing validation. Editorial boards should be explicit about the form and the level of validation they provide for papers and make this information plain to all users.

10. Statistics. Electronic delivery of information has changed the nature of statistics available to assess the usage and the 'value' of academic literature. Gathering statistics from the Internet is notoriously complicated, and even those who are knowledgeable about the pitfalls can be inadvertently or intentionally misled. As librarians and other decision makers increasingly rely on web statistics (such as the number of hits, page accesses or downloads) it is important to be informed about the nature of such measurements and the difficulty in gathering and interpreting them. Moreover, the value of a particular resource is often not best measured by simply counting the number of times it is currently used in some

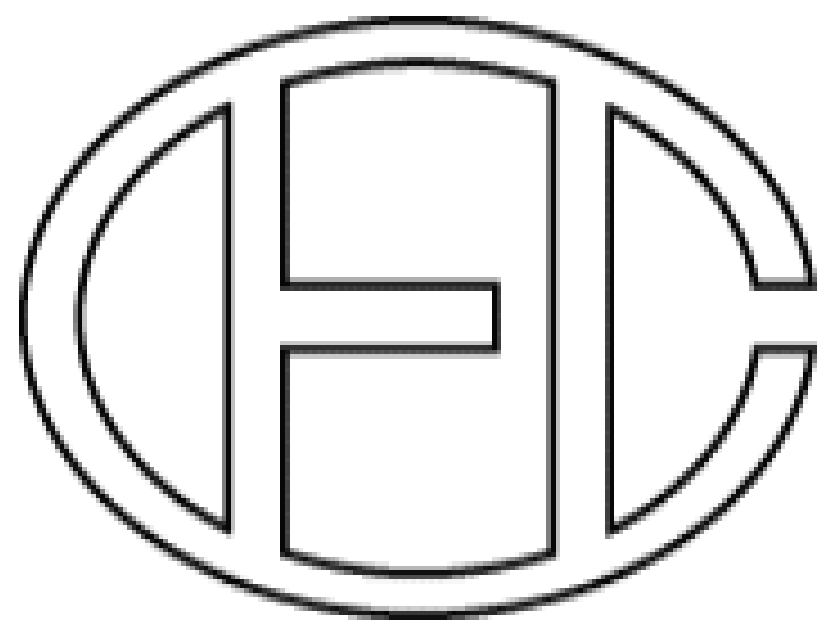

way. This is especially true in a field like mathematics in which current research continues to play such a significant role far into the future.

Given that statistics, while subject to misuse, are valuable and will be used, it is important that mathematics researchers and research librarians are alert to these rapidly changing issues and are prepared to make appropriate arguments for mathematics.

\section{For publishers and mathematicians}

11. Partial Access. Many journals restrict access to (paying) subscribers. As the web of mathematical literature grows, however, it will be increasingly important for all mathematicians to navigate that web, whether or not they have access to complete articles. This allows mathematicians to learn basic information about an article, even when they do not belong to institutions that have the financial resources to support the journal. It is especially advantageous to mathematicians from the developing world.

Journals should provide unrestricted access to tables of contents, abstracts of papers, and other data, such as keywords. Where practical, journals should also provide unrestricted access to reference lists with links, allowing all mathematicians to navigate the web of literature, even when they don't have access to the full-text of some parts of that web.

12. Eventual Free ACCess. The scholarly enterprise rests on the free exchange of ideas, and scholars need to have easy access to those ideas. Many journals, however, rely on subscriptions to recover costs and to provide an incentive to publish, forcing them to limit access to subscribers. Access should be a balance between those two needs, of scholars and of publishers. 
Limiting access to subscribers for a fixed period of time after publication may be necessary for many journals. In order to ensure appropriate accessibility for the electronic literature, we encourage all journals to grant free access after that fixed period of time.

13. ARChIVIng FORMAT. Ensuring the success of long-term archiving is more than storing the electronic data on reliable media in multiple locations. As software and formats change in the future, the data will require modification and updating. Not all electronic formats are suitable for these purposes.

In general, electronic documents should be stored in their most primitive format, that is, the format used to derive subsequent formats. Any format in which material is stored should follow an "open standard" that has a detailed public specification. This will increase the likelihood that scholars working decades or centuries from now will be able to use the material.

14. Archiving Responsibility. Traditionally, maintaining the older literature has been the responsibility of librarians rather than publishers. Even in the electronic age, scholars and the librarians who represent them have the greatest motivation among all of the affected parties to ensure the preservation of older material.

We recommend that electronic archives of the mathematical literature should ultimately be under the control of the academic community.

15. Licensing And Bundling. Some licensing and bundling arrangements for journals accelerate the transfer of control of our literature away from mathematicians and research librarians. When institutions are forced to accept or reject large collections of scholarly literature covering many different disciplines, the decisions are less likely to be made by scholars. As a consequence, the normal processes that promote the highest quality journals become less effective.

REMARK: The above recommendations have been stated in very general form. Whenever reference to existing formats [e.g., $\mathrm{AT}_{\mathrm{E}} \mathrm{X}, \mathrm{PDF}$ ], to archiving systems [e.g., arXiv], or to information and communication systems [e.g., Math-Net] has been made this is meant for illustration and not to promote these formats and systems. The IMU EC has asked CEIC to
The best protection, as always, comes through staying well informed and alert to these issues. In general, decisions about journal adoptions and cancellations should be made by academics and librarians.

\section{Postscript on Developing Countries.}

Today, active mathematicians depend on access to electronic information - online journals, databases of reviews, and preprint servers. More than access, research mathematicians need the tools to create and edit documents in standard formats [such as $\mathrm{LT}_{\mathrm{E} X} \mathrm{X}$, Postscript, and PDF]. This is true for mathematicians everywhere, including those in developing countries. Implementing many of the recommendations in the preceding document makes little sense if mathematicians are not connected to the Internet or have no tools to create electronic documents. National mathematical societies and academies in developing countries need to impress on their governments the need to establish the infrastructure necessary to provide high speed connectivity among academic institutions. The entire mathematics community should encourage and support specific actions designed to help in this effort, which include:

1. Establishing "mirror" services that provide quick access to users of electronic services within each region.

2. Establishing local help and service centers that spread expertise on the use of common standards [for example, $\mathrm{LT}_{\mathrm{EX}}$ ].

3. Creating small groups who tour the region and demonstrate the use of technology for research and study. Because scholarly communication is changing rapidly, there is great urgency to begin these efforts.

\section{Committee on Electronic Information Communication International Mathematical Union}

enhance, whenever appropriate and useful, individual recommendations by adding links to web pages that explain some of the technical issues involved, provide additional information, or contain (possibly controversial) discussions of the topics addressed. These links will be under the responsibility of CEIC and are not subject of the IMU EC recommendations.

Weitere Informationen:

Prof. Dr. Martin Gr"otschel

Konrad-Zuse-Zentrum f"ur Informationstechnik

Takustra"se 7

14195 Berlin

groetschel@zib.de 